\title{
Optimal Power Scheduling Scheme for Adhoc Network
}

\author{
Bharati V. Wukkadada, PhD \\ K J Somaiya's SIMSR \\ Vidya Vihar, Mumbai
}

\author{
Gopakumaran T. Thampi, PhD \\ Thadomal Sahani Engineering College \\ Bandra, Mumbai
}

\begin{abstract}
Power in Adhoc network is a constraint resource. Due to the node standalone characteristic, such networks are limited in performance under high dynamicity. In the process of power utilization, it is required to control its usage to improve network lifetime. To achieve this objective a power scheduling scheme is outlined in this paper. The proposed power scheduling scheme controls the node operability by scheduling a node link period, by the incorporation of node, network and refreshment mechanism. The proposed scheduling scheme, save the power utilization, retaining the data integrity by collaboratively operating in the whole network.
\end{abstract}

\section{Keywords}

Power scheduling, Adhoc network, network performance

\section{INTRODUCTION}

The up and coming technology in the wireless mode communication is ad-hoc network. The ad-hoc network encompasses a special subgroup of wireless networks as they have-not require the existence of centralized messages passing units. The wireless network simply required a fixed base station (BS) and it is responsible for routing messages from and to mobile nodes (MN) by the specified communication transmission range. But on the different side mobile ad-hoc network, do not require a single device other than the few mobile nodes which will be willingly to work co-operatively to form a network. The network will not depend on any BS, it will coordinate the messages to each and every mobile nodes. The individual nodes will form their own network and it will receive and forward the packets from and to each other nodes. This types of the special behavior will make the network created and work under the any adversarial conditions. There is an important matter in this strategy of wireless networks is exceptionally restricted limited energy supply of the nodes. But there are many different methods of energy-conservation protocols which have been developed for this types of networks [14], but the concept of energy problem is the one of the greatest problem here. So the restricted limited energy capacity of mobile devices are brought energy conservation to the forefront to concerns the enabling the mobile computing. It is depend on the battery life energy, it will consume the maximum that to achieve higher the network life. The conservation of the energy-resource in the ad-hoc network are various energy conservation protocol approaches by the many researches early. Of all the protocol approaches the communication scheduling approach is observed and for the optimal, as it is control the operational controlled purposes. Therefore it will give less demand for the device altering. The scheduling based communication area where the controlled switched devices are enabled and the routing based controlling will be proposed. Thus the scheduling based coding is in demand as mandatory as first for data accuracy. In an various situations it will be observed that when scheduling is done the energy resources information will be communicated through the different mobile nodes and the check the probability of data-lost occur and datafragmentation increases. Therefore it is required a a well energy scheduled- based- coding energy conservation for information accuracy will be recorded and maintained in adhoc networks.

\section{POWER SCHEDULING SCHEME}

In the mobile ad-hoc networking the very important challenging topic is how to minimize the consumption of energy and wireless network interface is usually a single biggest power consumer is devices. Hence the network is interface will often be idle, turn off the radio when it is not used, will be saving the power of the node. In reality this procedure is will not work out as practically. Where the node wants to communicate like send or receive a packet, the node cannot turn on and off arrangements. So the node wants to participate in any of the other situation like routing and control the protocols. In this situation it required a cooperation for power saving and the routing protocols is mainly required phenomenon for the ad-hoc network for the multihop ad-hoc wireless networks. In ad-hoc network the nodes must be forward the packets among each other for the healthier communication. In wireless ad-hoc, the topology management will decides the following for the each and every nodes behaviors:

1. Topology management decides which node will be turn on

2. Topology management decides when it should be turn on

3. Topology management decides at what transmit power

Under the first type of the topology management, the power off and on scheduling schemes some of the nodes are very rich in power, will be selected as that area links and the heads for the identification. Whereas these area head nodes will be selecting distributives in the network that each and every node area head is connected for the transmission to the area head. Further the gateway nodes will be selected as they forward pakcets among the area heads. The link will be formed a structure for routing a back- bone in ad- hoc network will be made by the area head virtually. Researchers have proposed many power off and on scheduling management for the topology schemes are SPAN, TMPO, CBTM, K-NEIGH, HDS etc.

In a Scheduling Priority switched port analyzer $\{$ SPAN\} topology management, the some of the co-coordinator nodes are selected in a manner as distributively. That they are away that two of the coordinators neighbors will not be reach each other directly or even via one or two coordinators. So the selecting rule confirms the communication connectivity for the ad-hoc network. This SPAN uses the power saving mode 
by the 802.11 standard because it will have the very high overhead broadcasting.

In Topology Management by Priority Ordering [TMPO], it will allocates inclinations and willingness value to each node, positioned on the energy level and speed of the node. If a node has very high inclination value then selected as aea head with the high probability. So topology of this type the power saving is also done via numerous different type of topology management schemes. And it will be based on the distribution of the each nodes in the network has power planning is carried out appropriately.

Each and every node in Cone-Based Distributed Topology Management [CBTM] will tries to find the least diffusing power $\mathrm{p}$ which will be diffuses with ' $\mathrm{p}$ '. Confirms that every cone of grade unit from place to place of each node and In this type we have check at least one neighbor node must be there.

Where as in the K-neigh type, each node will be adjusts its diffusing power, so it has $\mathrm{k}$ or slightly less than $\mathrm{k}$ one hop neighbors. This type of network transmission connectivity will be maintained as a condition for mobility purpose. Many of the algorithms are introduces for topology management are following first two methods or it will follow the third method. 1 step is switching among the active transmit, receive or idle. 2 step is then wakeup mode. 3 step is adjusting the transmission power, which may off and on power scheduling algorithms.

Whereas in Hop based Disjoint Scheduling [HDS] is an integrated model of power scheduling with the topology for network. The controlling and the scheduling will be supported by the three technique prerequisite and declaration steps and this will be sketched and method will be elucidated as below:

The qualification stage is defines as 3 stages:

- ACTIVE - QUALIFICATION-1: If no node is found active in its neighborhood area is activate the node

- ACTIVE - QUALIFICATION-2: The node will be activates itself when non-active upstream situation when the message get as select-msg from the active node.

- SLEEP QUALIFICATION: When the node is active neighbor node and no upstream information will be communicated.

Here at the time of announcement step, the node will be accomplished the following 3 procedures:

ACTIVE - ANNOUNCEMENT-1: If a sleeping node make its status as LISTEN and it will turns on its radio as well as it will try to find it is on or not. Under this situation where the node will be switches as ACTIVE state except if it fulfills the two verifications, firstly verification is Activequlaification- 1 is fulfilled and more than one node satisfies the qualification, if the process is selected again when a delay is offered. Secondly verification is the node will be in the listen phase after the active communication phase. After this it will further go to active qualification process, then it will take the phase as sleep mode.

ACTIVE - ANNOUNCEMENT-2: If the ACTIVE phase is switching, then the node will checks any neighbor as a lively active stream. In case if not then the active node will unicast it to SELECT - MSG to its active stream neighbors. It will select the node having energy, a HELLO- MSG will be sent by the node for activation count by the neighbors. The LISTEN phase will make a node to receive a SELECT - MSG will make to switches to its phase to ADDITIONAL ACTIVE phase mode. In case where the failing by the up sream neighbor due to a physical damage or any natural calamities, the active-node will be broadcasting as HELLO MSG for the purpose as those neighbor nodes takes part as transmitting node. A neighbor node which is active node then it will sends an ACK - MSG, after this the active node will selects any one because it will have most energy as transmitting node among all.

WITHDRAWAL ANNOUNCEMENT: the node will be selected as active node when it will give fair chance being a selection, each node will be stayed a ACTIVE for a definite interval of period tA at extreme. So this definite interval of period tA is resolute as reliant on the left over energy at individual node. The active node checks intermittently it remaining energy and it will change its state as WITHDRAWAL at the period where remaining energy dashes some edge value. Withdrawing neighbor node is preserved as sleeping node for the similar period tL. The node will checks for the sleep - qualifications again after the tL. Under the condition where the sleep - qualifications is fulfilled, then it will go the state as SLEEP phase. Else it will change as back to its original or previous phase. Here following figure will depict the state transitions diagram for the wakeup scheduling for the hop based disjoint.

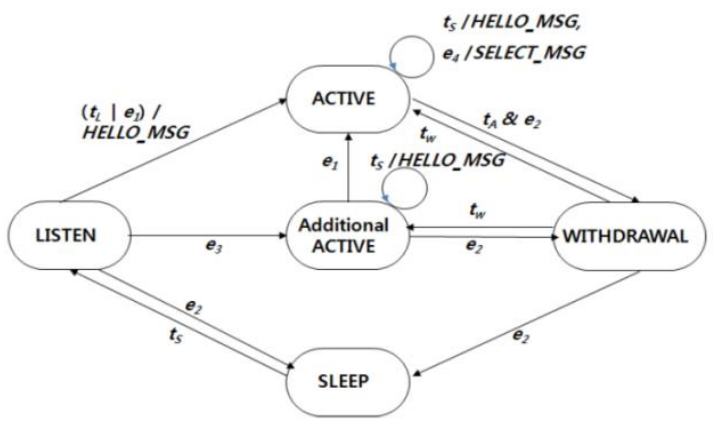

Figure 1: Status transition diagram of the Hop Based Disjoint wakeup scheduling [1]

The approach to energy conservation for the density based wireless ad-hoc network, where this scheme is further enhanced and optimized by considering the characteristics for the delivery for the network. Here the scheduling scheme will not consider any type of network parameters and standalone controlled working. The concept the energy consumption based on network topology delivery will be further act as save the power consumption and improving life-time of the network. Like these types of approaches were approached earlier like SPAN and TMPO. To improve the scheduling approach, a topology scheduling tool of active scheduling is done.

\section{TOPOLOGY DRIVEN POWER SCHEDULING APPROACH}

The projected style of approach is industrialized with the consideration of three basic processes for HDS is used here for the combination of topology features will be under contemplations. For a distribute network with $\mathrm{N}_{\mathrm{i}}$ nodes, $\mathrm{L}_{\mathrm{i}, \mathrm{k}}$ 
links and $\mathrm{P}_{\mathrm{i}, \mathrm{N}}$ powers per node, a power optimization scheme is proposed. In the first stage of operation, the Hello packet transmission is used to discover a one Hop neighbor. These nodes formulate a neighbor list with basic node information's, such as their Neighbor nodes, node power, transmission range and available data rate. One the creation of neighbor tables, a search algorithm is executed to formulate a 1-hop neighbor group, which is termed as 1-NG. Similar K, 1-NG groups were created and for each 1-NG a junction node is obtained. The Junction node $\mathrm{J}_{\mathrm{i}}$ is defined as a node having its proximity for 2, 1-NG groups. With this this initialization steps the power scheduling approach is executed to conserve the power utilization. To perform the operation, 3 operational stages are outlined defined as;

\section{Active_Master selection \\ 2. Upstream Link selection \\ 3. Wake up scheduling}

\section{ACTIVE_MASTER SELECTION}

Active_Masters along with Links endorse virtual backbone by the path, where a demand for aditonal transmission of power and is used for routing, reception and processing of packets. Consequently the Active_Masters should be selected in such a way that they have enough/higher power level. For the selection process of Active_Master, a selection algorithm is executed,

\section{Algorithm 1:}

\section{Active_Master Selection (Executed at Initialization process)}

For each 1-NG group, a Active Master is selected. This selection is carried out with broadcasting of each power level to its neighbor. A selection process is then carried out as outlined below,

Initialize the current node power $\left(\mathrm{P}_{\mathrm{i}}\right)$ as Peak_power $\left(\mathrm{MP}_{\mathrm{i}}\right)$

$\mathrm{MP}_{\mathrm{i}}=\max \left(\mathrm{P}_{\mathrm{i}}\right)_{1-\mathrm{NG}}$

for Each neighbor node $\mathrm{N}_{\mathrm{i}}$ do,

if power of node $\mathrm{N}_{\mathrm{i}}, \mathrm{P}_{\mathrm{i}}<\mathrm{MP}_{\mathrm{i}}$ then

Node_Mode $=$ Active;

else

if Power of $\mathrm{N}_{\mathrm{i}}>\mathrm{MP}_{\mathrm{i}}$, then

$\mathrm{MP}_{\mathrm{i}}=\mathrm{P}_{\mathrm{i}}$

End

Nodes periodically checks among its one hop neighbors and if a node has $\mathrm{MP}_{\mathrm{i}}$ among such one hop neighbors, it declares itself as a Active_Master. If participating node knows that its neighbor node has become Active_Master from received broadcast message, it changes it's status to Active. It declares its status as Active and it's current Active_Master in next broadcast message. If more than one neighbors of an participating node became active_master, participating node select its active_master node from which it has received the broadcast packet earlier.

There may be participating nodes whose one hop neighbors with power level more than the participating node chose to join Active_Masters, as the Active_Masters have more power level than its one hop neighbors. Such participating nodes with maximum power level among one hop participating neighbors declare themselves as Active_Masters in the next broadcast message. A Active_Master prepares a list of its Active nodes, which are joined to the Active_Master, form the broadcast of broadcast messages received from one hop neighbors. This information in the table is periodically changes as a new broadcast packet is received. The Active_Master will drain its energy more rapidly, as compared to Active nodes. Before the Active_Master loses its major part of its power, the responsibilities of the Active_Master should be transferred to other node with sufficient power level. Also Active_Masters should not be changed frequently which will increase the overhead. When a Active_Master observes that its power level is gone below a threshold, it will withdraw its status of Active_Master. The withdrawal of Active_Master is declared to its Active nodes in the next broadcast message as a participating node. The threshold is set to $80 \%$ of power level when the node decided to become a Active_Master. When a junction Node or Active node comes to know that it cannot contact its Active_Master, it changes its status to participating and starts Active_Master placement procedure.

\section{UPSTREAM LINK SELECTION}

There are 2 maximum hops will be there for the any 2 close active_masters, so the required links will be required and are forwarded option will be used for the packets exchange between the active_masters. The Link nodes must have sufficient amount of power, to transmit and receive the packets to and from the Active_Masters.

\section{Algorithm 2: \\ (Link Selection, executed by Active_Masters)}

In each $1-N G$ group, the broadcast packets 'AWAKE' is transmitted,

Wait for one link period

for Each Active_Master within two hops do

If Active_Master has not decided Link for this Active_Master then

If Active_Master is not neighbor of my existing Links, then

Link $=$ Active which has maximum power among neighbors of Active_Master

else

Active_Master . Link $=$ my existing Link

end if

else

Active_Master. Link $=$ Link of Active_Master for this node end if

end for

To determine the Links, Active_Masters needs information in its two hop neighborhood. This information is obtained from the broadcast packets; it has received from its one hop neighbors. But as the Active of different Active_Masters are not synchronized, they may miss the broadcast packets from Actives of different Active_Masters. Active_Master periodically sends broadcast request packet AWAKE to its Actives to put them in alisten mode for at least one link period. Active_Master finds out all the Active_Masters within two hops and Active_Master selects its Active as a Link which has maximum power level, for each Active_Master within two hops. Generally the Link is taken such that it has more number of neighbors to ensure less number of Links. If any Active_Master within two hops have already declared their Links, then there is no need to select Link for such Active_Master. The Active_Master determine the validity of the Link by node. Periodically $p$ ower level below the threshold level then starts the selection procedure for new Link by the Active_Master.

\section{WAKEUP SCHEDULING}

Here the features of 802.11 CSMA / CA for power saving for further for the MAC power layer randomized by the 
efficiently listen up time for Active nodes in ad hoc network is proposed. Active_Masters and Links continuously AWAKE to forward packets of other nodes. Active nodes listen up a number of times a period $\mathrm{T}$ in a link, as well as they will goes off again that when it is idle or there is no data to transmit or receiving.. There are number of Wakeup cycle periods $\left(\mathrm{T}_{1}\right.$, $\left.\mathrm{T}_{2}\right),\left(\mathrm{T}_{2}, \mathrm{~T}_{3}\right) \ldots\left(\mathrm{T}_{\mathrm{n}}, \mathrm{T}\right)$ in a link period. Active nodes listens up once in a Wakeup cycle. All nodes stays alisten during period $\left(0, \mathrm{~T}_{1}\right)$ called as broadcast window to exchange broadcast packets. Each node synchronizes its clock by using time stamp of broadcast message from Active_Master. Each Active node determines its listen up time from its node id and a Listen counter $\mathrm{w}_{\mathrm{i}}$ given below;

\section{Algorithm 3:}

(Wakeup cycle scheduling, executed by all Active nodes)

Transmit broadcast packet at appropriate time in $\left(\mathrm{T}, \mathrm{T}_{1}\right)$

For Each Wakeup cycle period $\left(\mathrm{T}_{\mathrm{m}}, \mathrm{T}_{\mathrm{m}+1}\right), \mathrm{m}=1 \ldots \mathrm{n}$, do

if there are no packets to transmit then

Go to Wakeup mode until time $t_{\text {im }}$

Remain in listen up mode until time $t_{i m}+t$

if Packet received at time in $\left(t_{i m}, t_{i m}+t\right)$ then

Remain in listen up mode

end if

if time $<\mathrm{T}_{\mathrm{m}+1}-\mathrm{T}$ then

Go to Wakeup mode until time $\mathrm{T}_{\mathrm{m}+1}$ end if

else

Transmit the packet (s) at appropriate time(s)

During the initial period $\left(0, \mathrm{~T}_{1}\right)$ of the link period $(0, \mathrm{~T})$, all node remain a listen, transmit broadcast messages if any. And link messages so as to keep every node undated about one hop neighborhood. We call the period $\left(0, \mathrm{~T}_{1}\right)$ as broadcast window. Thus when a packet, other than broadcast and broadcast, comes at MAC layer for transmission during broadcast window, the packet can not be sent immediately. The packet has to be buffered at the MAC layer and it will be transmitted after the end of broad cast window. At the end of broadcast window, i.e., at time $T_{1}$, all Active nodes go to Wakeup mode, if the node donot have any packets for transmission. Each Active node with $\mathrm{N}_{\mathrm{i}}$ listens up at time $\mathrm{t}_{\mathrm{im}}$ in $\mathrm{m}^{\text {th }}$ Wakeup cycle. Node $\mathrm{N}_{\mathrm{i}}$ calculates its listen up time $t_{\mathrm{im}}$ for a pseudo-random number with its node $\mathrm{N}_{\mathrm{i}}$ and a Listen counter $\mathrm{w}_{\mathrm{i}}$ as seed to the pseudo-random number generator. Listen up time of a node $N_{i}$ is given by $t_{i m}$.

$\mathrm{t}_{\mathrm{im}}=\mathrm{T}_{\mathrm{m}}+\left(\mathrm{T}_{\mathrm{m}+1}-\mathrm{T}_{\mathrm{m}}\right) * \mathrm{R}_{\mathrm{an}}$

Where $\mathrm{R}_{\mathrm{an}}\left(\mathrm{i} \mathrm{x} \mathrm{w}_{\mathrm{i}}\right)$ is pseudo-random number in $(0,1)$ with

$\mathrm{i} * \mathrm{w}_{\mathrm{i}}$ as seed.

This listen up time $t_{\text {im }}$ is also known to Active_Master and one hop neighbors of node I as each node knows its one hop neighbor id and its Listen counter wi from broadcast packet. All the nodes in ad hoc network have identical pseudorandom number generator. So when a Active_Master or neighbor of node I wants to send packets to $\mathrm{N}_{\mathrm{i}}$, it will send at time $t_{i m}$. After the node $N_{i}$ receive packets, it goes to off again. So for Active_Master and neighbors of node I, the packets have to be buffered at MAC layer until time $t_{\mathrm{im}}$. After a small time $t_{i m}$, if no packets are send to it, it goes to off again. If the node $\mathrm{N}_{\mathrm{i}}$ wants to send packet to the Active_Master, it senses the channel from the end of broadcast window or when arrives at MAC layer for transmission, until the channel is idle. The node $\mathrm{Ni}$ uses standard 802.11 back-off algorithm, if contention for channel occurs.

When the channel is idle, it will send packets to the Active_Master. Node Ni Wakeup after transmission is over and listens up at time ti or T, whichever comes earlier. If the node $\mathrm{k}$ wants to send packets to its neighbor $\mathrm{Ni}$ other than its Active_Master, node $\mathrm{k}$ listens up at time $\mathrm{t}_{\mathrm{i}}$ as node $\mathrm{N}_{\mathrm{i}}$ listens at time $t_{\mathrm{i}}$, and send packets to node $\mathrm{N}_{\mathrm{i}}$, if the channel is idle. After each Wakeup cycle $(\mathrm{Tm}, \mathrm{Tm}+1)$ in link period, Listen counter wi increased. If wi was not changed, the node $\mathrm{k}$ with listen up time $t_{\mathrm{km}}$, little earlier than listen up time tim of node $\mathrm{Ni}$ will get more throughput than node $\mathrm{Ni}$, as packets transmitted to node $\mathrm{k}$ will always overlap to the listen up period $\left(t_{i m}, t_{i m}+T\right)$ of node $N_{i}$. Thus node $k$ will get more throughput than node $\mathrm{N}_{\mathrm{i}}$. When the Listen counter $\mathrm{w}_{\mathrm{i}}$ is increased after every Wakeup cycle $\left(\mathrm{T}_{\mathrm{m}}, \mathrm{T}_{\mathrm{m}+1}\right)$ in link period $\mathrm{T}$, listen up time of all nodes are redistributed in the time period $\left(\mathrm{T}_{\mathrm{m}}, \mathrm{T}_{\mathrm{m}+1}\right)$ and all Active nodes get fair share of throughput.

In Ad hoc networks, traffic passing through the backbone nodes, i.e., Active_Masters and Link nodes is expected to be large as compared to local traffic between neighboring nodes. Active_Masters and Links do not have to wait for longer time for idle channel. Thus the overall delay for routing packets will be reduced. Whereas, if the packets are to be transmitted to the neighbors, sender has to wait until the listen up time of receiver. Thus the delay for local traffic is expected to be more.On average each packet suffers delay slightly more than $\left(\mathrm{T}_{\mathrm{m}}-\mathrm{T}_{\mathrm{m}+1}\right) / 2$ at last hop. To reduce this delay, the number of Wakeup cycles can be increased. This will reduce the delay at last hop, as sender has to wait for less amount of time to deliver the packet to receiver at last hop.

\section{PERFORMANCE EVALUATION}

For the evaluation of the proposed scheduling scheme, a randomly distributed network is considered. The nodes are haphazardly dispersed over a $\mathrm{n} \times \mathrm{n}$ area, and each node is deliberated to have a transmitter and receiver unit with the energy consumption model of IEEE 802.11 high rate DS operating at $2 \mathrm{mbps}$ for the network interface card (NIC). The Power consumption units for the operation of transmission, reception and wakeup period is as defined in table 1 .

\begin{tabular}{|l|l|l|l|}
\hline Tx & Rx & Idle & Wakeuping \\
\hline $1400 \mathrm{~mW}$ & $1000 \mathrm{~mW}$ & $830 \mathrm{~mW}$ & $130 \mathrm{~mW}$ \\
\hline
\end{tabular}

Table1: Power consumption in various modes For the develop protocol the performance were evaluated with the following network parameter.

For the generation of the network topology the considered network characteristic is as outlined in table 2.

Table 2: Network Parameters

\begin{tabular}{|l|l|}
\hline Network parameter & Characteristic \\
\hline Node density $\left(\mathrm{N}_{\mathrm{d}}\right)$ & 30,45 \\
\hline Network Area & $\mathrm{N}_{\mathrm{d}} \times \mathrm{N}_{\mathrm{d}}$ \\
\hline Communication range & 80 Units \\
\hline Mobility & Non-static \\
\hline
\end{tabular}




\begin{tabular}{|l|l|}
\hline Topology & Random \\
\hline MAC & 802.11 \\
\hline Power model & $\begin{array}{l}\text { IEEE 802.11- } \\
\text { NIC card }\end{array}$ \\
\hline
\end{tabular}

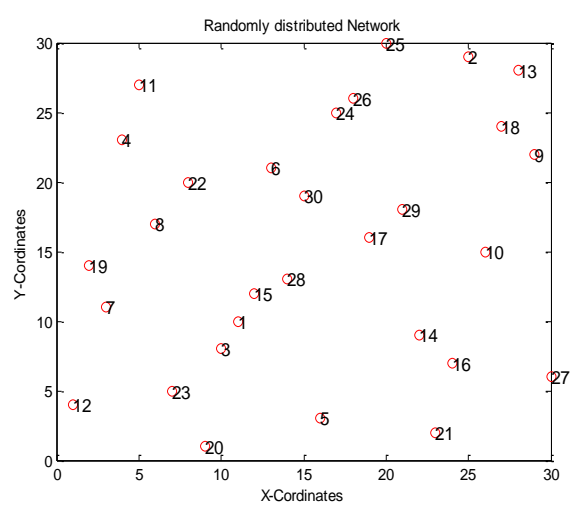

Figure 2: Random Distributed Network topology

To evaluate the proposed approach, a randomly distributed network topology is considered. The nodes are defined as a randomly distributed units with there $\mathrm{x}$ and $\mathrm{y}$ coordinates varying. These variations are in synonymous to the geographical position of a node in real time. The randomly scattered nodes used for the simulation is as shown in figure .2

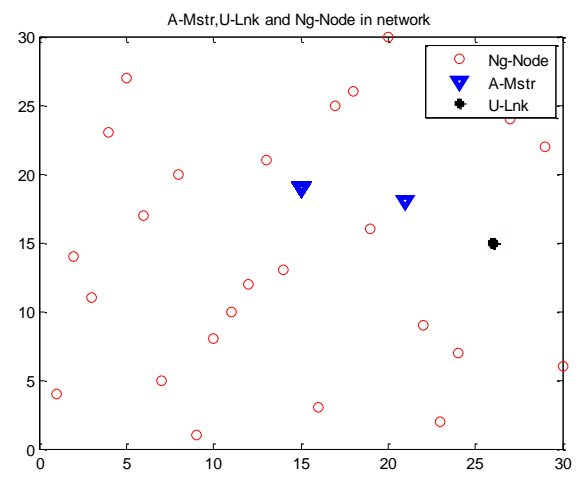

Figure 3: Active Master and uplink nodes

Over the developed network, a routing algorithm based on ondemand routing scheme is proposed. For the generated routes a topology driven power scheduling approach is made. For the development of the proposed approach, the nodes are iterated over $\mathrm{N}$ nodes and an active master nodes is found. To the found active master nodes uplink nodes are defined, over which the proposed topology driven power scheduling algorithm of executed. The obtained Master, uplink and neighbor nodes are as outlined in figure 3 .

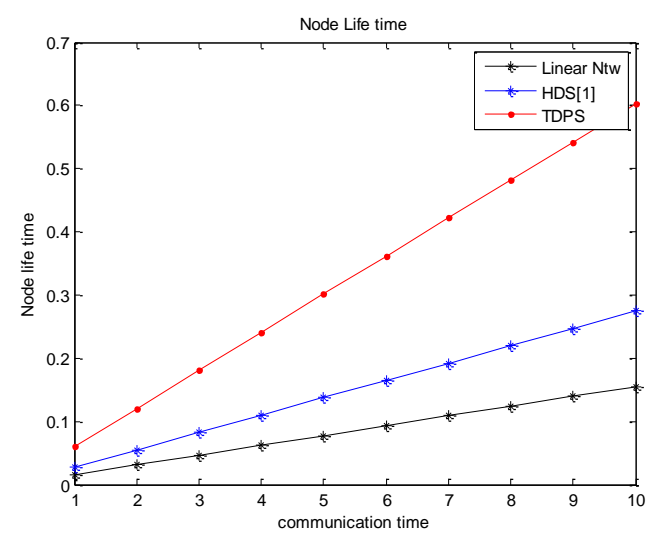

Figure 4: Node life time over range of communication period

For the proposed scheduling algorithm, a evaluation is carried out for node life time. It is observed that, as the communication time period elapse, the node life time is observed to be diminished for linear network. A linear network is defined as a network with no optimization scheme running. Here the packets are forwarded on a linear forwarding manner. It is observed that the nodes are very quickly shut down due to abrupt power dissipation. However with the utilization of HDS [1], the power consumption is controlled, hence the node life time is improved. This parameter is further improved by consideration of nodes aligned in the network. The node distribution pattern is considered and in accordance a scheduling algorithm is developed which saves higher power, giving longer node life time.

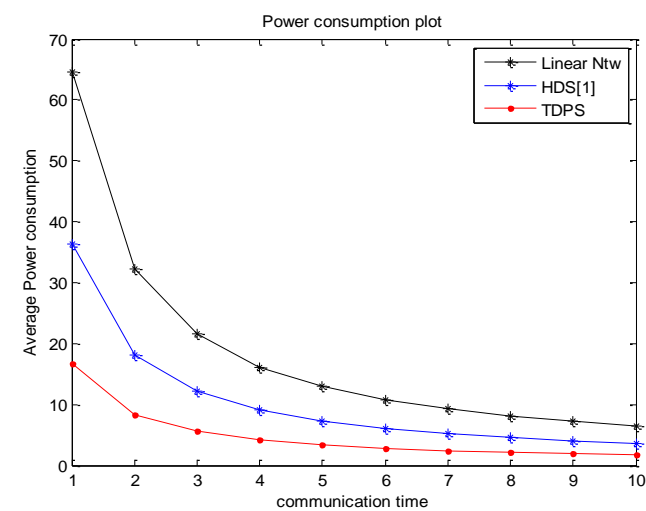

Figure 5: Average power consumption

The average power consumption model is observed in figure 5. The power consumption of the three methods are evaluated. It is observed that, the power for the linear model is quite higher than the other two methods. However with the elapse of compunction time period, the power consumption get reduced, as in linear topology. The power is almost drained out, and where as in the other two algorithms the power are saved to improve network life time 


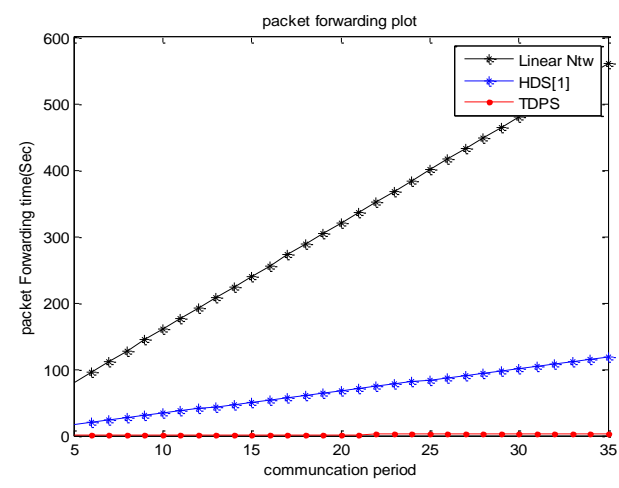

Figure 6: Packet forwarding time plot

As the proposed scheduling scheme, controls the communication time period, the overall communication period is observed to be very low in case of the proposed TDPS over other two technique. As the scheduling algorithm wakeup the node for a limited period to transmits or receive data and majority of the communication period the node is kept low, the power saved is higher. As well the packets are forwarded via master nodes, the packets are forwarded even when the individual node is at low. Hence there is not buffering of data, and hence the time taken is low. Whereas in case of linear of HDS the packets are either be kept on queue, or in retransmission state if a link drains out. This increases the packet forwarding time in the network.

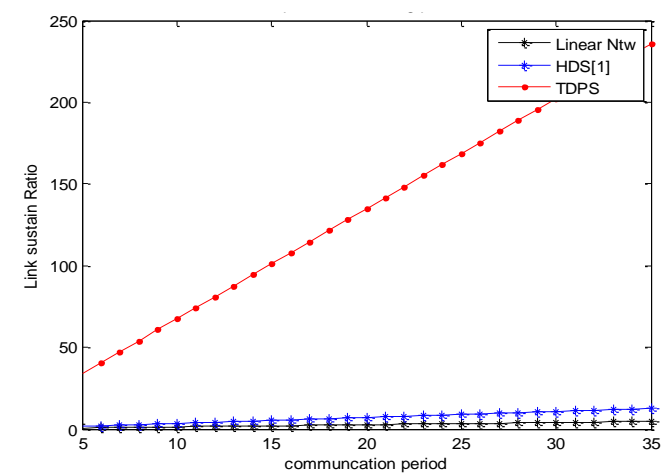

Figure 7: Link sustain plot for the random network

With the analysis, the link sustaining ratio is evaluated. The link sustaining time $\mathrm{s}$ defined as the amount of power consumed over a communication period for the total nodes present. It is observed that sustain time of the links are higher in case of proposed TDPS approach. The approach raises the link sustain time, because the power consumption per node is very low, as they are kept at low, and also the active communication period is low in such case. To evaluate the effect of variation in node density a similar analysis is performed over a higher density nodes with node density $\mathrm{N}_{\mathrm{d}}=45$ and the observation has been illustrated figure 8 to 13 .

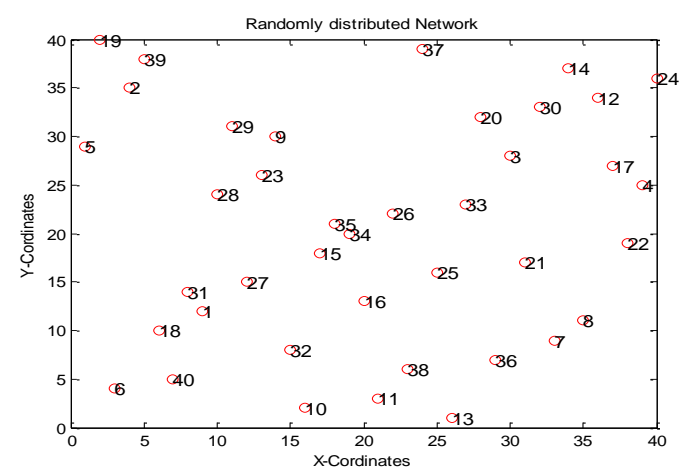

Figure 8: Network topology for Node density $(\mathrm{Nd}=45)$

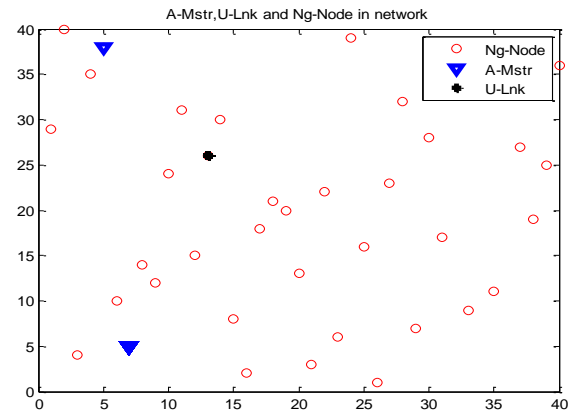

Figure 9: Selected nodes for communication at $\mathbf{N d}=45$

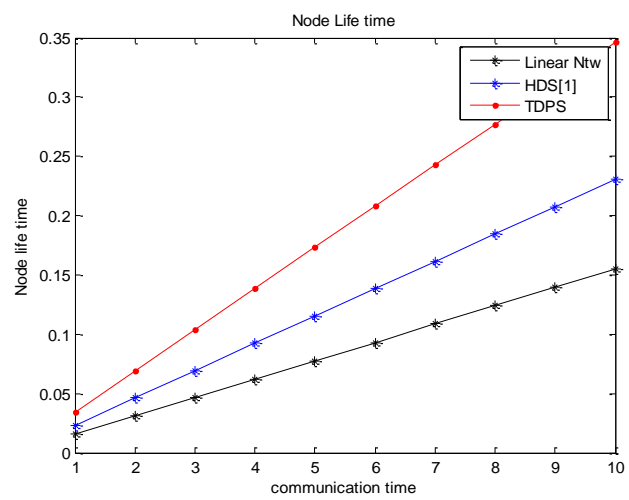

Figure 10: Node Life time at $\mathbf{N d}=45$

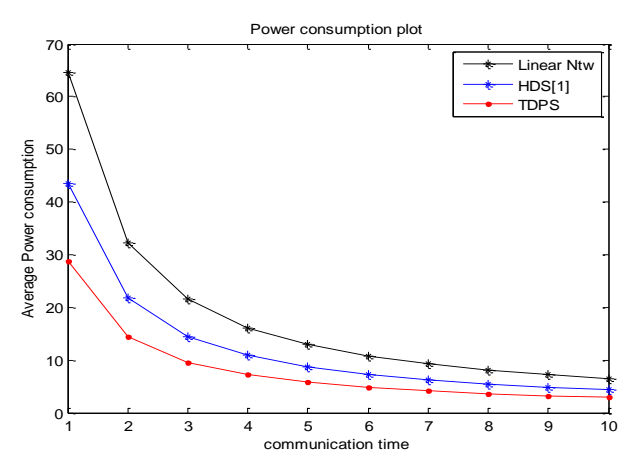

Figure 11: Power consumption at Node density, $\mathrm{Nd}=45$ 


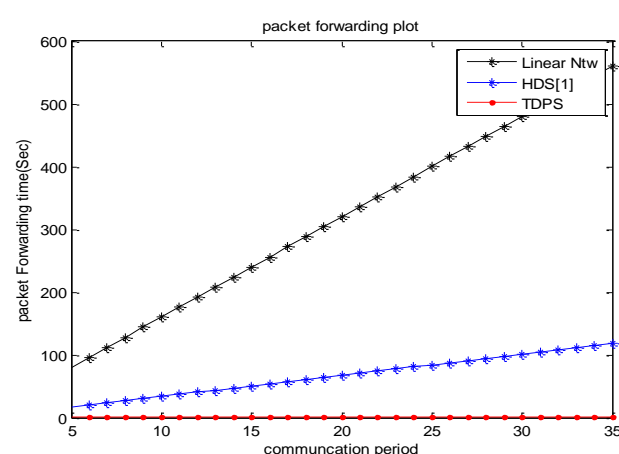

Figure 12: Packet forwarding time at $\mathrm{Nd}=45$

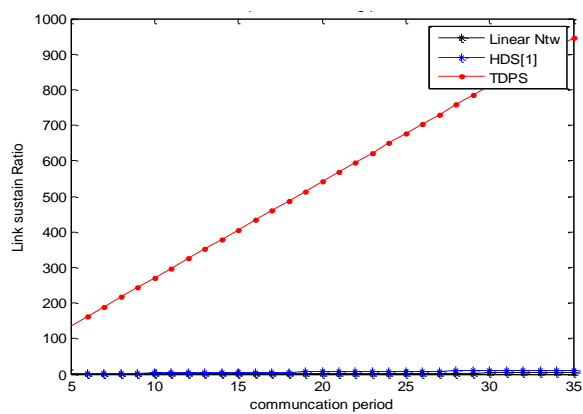

Figure 13: Link sustain ratio for $\mathrm{Nd}=45$

The evaluative factors are observed to be improved by using proposed TDPS approach, in the case of communication time period, the overall time is lowered, hence power consumption is also minimized. This improves the link sustaining time and as well node life time is increased.

\section{CONCLUSION}

Our research in this paper, we did presents a coding approach for power scheduling in wireless ad- hoc network. The approach of power scheduling is carried out by the consideration of network topology and node power level. In contrast to the conventional power scheduling approach wherein scheduling of power is carried out based on isolated nodes. In this research the node distribution and network architecture is considered and in concern to the neighboring node characteristic and power, scheduling is carried out. This mode of communication is observed to be more efficient in communication than the conventional flat topology approach, which follows a disjoint based wake up scheduling scheme.

\section{REFERENCES}

[1] EunHwa Kim. 2013 A Density Control Scheme Based on Disjoint Wakeup Scheduling in Wireless Sensor Networks, International Journal of Smart Home Vol.7, No.5,

[2] Gil Zussman, Adrian Segall. 2003. Energy efficient routing in ad hoc disaster recovery networks, Elsevier,

[3] Y. Xu, S. Bien, Y. Mori, J. Heidemann, and D. Estrin January2003 . Topology control protocols to conserve energy in wireless ad hoc networks, IEEE Transactions on Mobile Computing, Technical Report CENS Technical Report 0006, Center for bedded Networked Sensing,

[4] YaXu. July 2001. Geography informed Energy Conservation for Ad Hoc Routing, ACM/IEEE International Conference on Mobile Computing and Networking.

[5] J.H. Chang, L. Tassiulas. March 2000. Energy conserving routing in wireless ad-hoc networks, IEEE INFOCOM,.

[6] ImrichChlamtaca, Chiara Petriolib, Jason Redi. March, 1997. Analysis of Energy-Conserving Access Protocols for Wireless Identification Networks, (ITC-97),

[7] J.H. Chang, L. Tassiulas. September 1999. Routing for maximum system lifetime in wireless ad-hoc networks, $37^{\text {th }}$ Annual Conf. on Communication, Control, and Computing,

[8] ArunAvudainayagam, Wenjing Lou, and Yuguang Fang. 2003. DEAR: A Device and Energy Aware Routing protocol for heterogeneous ad hoc networks, Elsevier.

\section{AUTHOR'S PROFILE}

Dr. Bharati V Wukkadada is an Assistant Professor in Area -IT at K. J. Somaiya Institute of Management Studies and Research, Vidyavihar, Mumbai.

Dr. Gopakumaran T Thampi, is a principal of Thadomal Shahani Engineering College (TSEC), Bandra, Mumbai. 